\title{
LA SITUACIÓN DE LOS DERECHOS HUMANOS DEL PUEBLO RAPA NUI: EL DERECHO DE LIBRE DETERMINACIÓN Y LOS DERECHOS TERRITORIALES.
}

\author{
THE HUMAN RIGHTS SITUATION OF THE RAPA NUI \\ PEOPLE: THE RIGHT OF SELF-DETERMINATION AND \\ TERRITORIAL RIGHTS.
}

\section{LUÍSA BRANDÃO BÁRRIOS*}

\section{Resumen}

Una de las más graves vulneraciones a los derechos de los Rapa Nui, que se mantiene hasta la fecha, es la usurpación de su territorio, mediante la inscripción de la totalidad de la Isla de Pascua a nombre del Estado de Chile (1933), época en que los Rapa Nui eran considerados apátridas y carecían de todo derecho civil y político. Dicha inscripción se realizó en el Conservador de Bienes Raíces de Valparaíso, ciudad continental ubicada a más de 4.000 kilómetros de la isla, excluyendo cualquier posibilidad de oposición, utilizándose como argumento que la tierra carecería de dueño. A partir de la "Ley Pascua", se reconocen derechos de ciudadanía y otros beneficios a los Rapa Nui, lo que se refuerza con legislaciones posteriores como la "Ley Indígena" (Ley 19.253 DO 05.10.93), a principios de la década del 90 que otorga especiales derechos a los Rapa Nui y la ratificación del Convenio 169 de la OIT sobre Pueblos Indígenas y Tribales en Países Independientes. En la práctica, dicha legislación no se ha traducido en la devolución de la tierra y respeto a derechos territoriales y de libre determinación de los Rapa Nui. La situación de los derechos

Artículo recibido para su evaluación el 10 de octubre de 2021, y aprobado para su publicación el 30 de noviembre de 2021

*Doctora en Derecho, Universidad de Salamanca. Estudiante de Posdoctorado, Universidad de Salamanca. Email: luisabarrios@icloud.com 
humanos del Pueblo Rapa Nui y de sus demandas, con especial mención a los derechos territoriales y de libre determinación, es de suma importancia, bien como, la demanda de participación política efectiva y control sobre sus instituciones políticas por la vía del establecimiento de un "estatuto especial", de una modalidad de control migratorio y una especial referencia a los esfuerzos por lograr el cumplimiento del derecho a la consulta previa a los pueblos indígenas; los antecedentes con respecto a su demanda colectiva por recuperar su territorio ancestral, por el respeto a su derecho a la autodeterminación de acuerdo al derecho Internacional y por el pleno reconocimiento del Acuerdo de Voluntades de 1888. La situación de los derechos de los pueblos indígenas en Chile en general se destaca por la falta de reconocimiento constitucional, la inexistencia de un mecanismo oficial para la consulta previa en caso de medidas susceptibles de afectarles directamente o asegurar su participación política y la carencia de medidas claras para la implementación del Convenio 169 de la OIT vigente en Chile desde septiembre de 2009. Hasta la fecha no existe este reconocimiento constitucional, que surgirá con la reforma constitucional, a la que asistimos actualmente.

\title{
Palabras Clave
}

Rapa Nui; derecho a la tierra; derecho a la libre determinación; derechos humanos; Constitución.

\begin{abstract}
One of the most serious violations of the rights of the Rapa Nui, which continues to date, is the usurpation of their territory, through the registration of the entire Easter Island in the name of the state of Chile (1933), epoch in which the Rapa Nui were considered stateless and lacked all civil and political rights. The registration was made in the Real Estate Curator of Valparaíso, a continental city located more than 4,000 kilometers from the island, excluding any possibility of opposition, using as an argument that the land would lack an owner. As of the "Easter Law" (Ley 19.253 DO 05.10.93), citizenship rights and other benefits are recognized for the Rapa Nui, which is reinforced with subsequent legislation such as the "Indigenous Law" at the beginning of the 90s that grants special rights to the Rapa Nui, and the ratification of ILO Convention 169 on Indigenous and Tribal Peoples in Independent Countries. In practice, the
\end{abstract}


legislation has not resulted in the return of the land and respect for the territorial rights and self-determination of the Rapa Nui. The human rights situation of the Rapa Nui People and their demands, with special mention to territorial rights and self-determination, is of utmost importance, as well as the demand for effective political participation and control over their political institutions through the establishment of a "special statute", a form of Migration Control and a special reference to efforts to achieve compliance with the right to prior consultation with indigenous peoples; the antecedents with respect to their collective demand to recover their ancestral territory, for the respect of their right to self-determination in accordance with International law and for the full recognition of the Agreement of Will of 1888. The situation of the rights of Indigenous Peoples in Chile in general stands out for the lack of constitutional recognition, the inexistence of an official mechanism for prior consultation in the event of measures likely to affect them directly or ensure their political participation, and the lack of clear measures for the implementation of ILO Convention 169 in force in Chile since September 2009. To date, there is no such constitutional recognition, which will arise with the constitutional reform, which we are currently witnessing.

\section{Keywords}

Rapa Nui; right to land; right to self-determination; human rights; Constitution.

\section{BREVE RESEÑA HISTÓRICA.}

La historia de Rapa Nui, originalmente llamada Te pito o te henua, El ombligo de la Tierra o Mata ki te rayi, Ojos que miran al cielo, es inseparable de su condición remota.

Hacia el año 1.000, la sociedad de Rapa Nui alcanzó su máximo apogeo y experimentó un fuerte aumento demográfico, iniciándose la construcción de centros ceremoniales de culto a los antepasados, representados a través de gigantescas estatuas de piedra: los moais. En el contexto de una sociedad fuertemente estratificada, ordenada a través de linajes extensos que controlaban un determinado territorio, la construcción de altares a los antepasados y el levantamiento de los enormes moais, cumplían la función de reflejar el poder y cohesión interna de cada clan. El poder político estaba 
concentrado en el Ariki Mau, autoridad suprema de carácter hereditario, y en la casta sacerdotal, encargada de mantener las tradiciones religiosas y el culto a los antepasados.

En sus 128 años de historia con el Estado de Chile se han establecido diversas leyes y decretos, y se ha evaluado la adecuación de estas a las necesidades particulares de los habitantes de esta zona del océano Pacífico.

Desde su colonización inicial por inmigrantes polinesios, su extremo aislamiento favoreció el desarrollo de una cultura de rasgos únicos en el mundo, la que sólo ha podido ser reconstruida gracias al aporte de la arqueología y la etnología.

El crecimiento de la población, que se estima alcanzó a las 10.000 personas, hizo que la presión sobre los recursos y la competencia entre los distintos linajes, se hiciera más intensa. La situación llegó al límite cuando la deforestación casi total de la isla impidió construir embarcaciones que podrían haber aliviado la presión demográfica sobre alimentos insuficientes. Los requerimientos de la clase sacerdotal se hicieron cada vez más difíciles de cumplir, y el poder de los ancestros ya no fue suficiente para sostener la cohesión interna de los linajes y el delicado equilibrio social.

A mediados del siglo XVII, o quizás antes, la situación hizo crisis, estallando un feroz conflicto interno en el que la gran mayoría de los moais fueron destruidos por clanes rivales. El reacomodo a la nueva situación ambiental fue lento y difícil, y cristalizó en el culto a Make Make - el Dios creador - y en la ceremonia del tangata manu - hombre pájaro, en la que los distintos linajes competían anualmente por el poder político. El ganador asumía un carácter sagrado, debiendo vivir solo y aislado; mientras, su grupo adquiría un poder despótico sobre el resto de la población, que incluía sacrificios humanos a los dioses para así asegurar el bienestar para el año. Estas prácticas renovaron constantemente las hostilidades entre los grupos, produciendo un clima de permanente violencia y crisis social.

Durante el siglo XVIII visitaron la isla los primeros navegantes europeos, que fueron quienes dieron a conocer Rapa Nui ante el mundo.

En el siglo XIX, una serie de expediciones esclavistas y la llegada de europeos inescrupulosos, hicieron que la población se redujera al mínimo, víctima de la caza de esclavos y la viruela. Las jerarquías tradicionales se desmoronaron y la llegada de misioneros católicos a la isla reforzó la creciente aculturación. 
Bernardo O’Higgins Riquelme es considerado el padre de la patria. ${ }^{1}$ Su presencia, como militar y gobernante, fue crucial en todo el proceso de emancipación chilena del dominio español, había sido crucial luchando en las batallas de la Independencia o ejerciendo como primer Director Supremo de la nueva nación. Durante el período de la Reconquista, organizó en Mendoza, junto a José de San Martín, el Ejército Libertador de Los Andes y dirigió la ofensiva chilena. Derrotadas las fuerzas realistas, asumió como Director Supremo y firmó, el 12 de febrero de 1818, la Proclamación de la Independencia de Chile.

Sin embargo, su afán por incrementar el desarrollo económico del país, lo impulsó a establecer relaciones comerciales con las principales monarquías europeas; las cartas enviadas a los reyes, escritas en un lenguaje equilibrado y cauteloso, quedaron para la posteridad como un testimonio de su carácter eminentemente pragmático.

Años después, en 1868, la corbeta O’Higgins cumplió los deseos del Capitán General, puesto que aquella fue la primera expedición realizada por el Gobierno de Chile con el fin de incluir a Rapa Nui dentro de su soberanía.

En un artículo de 1885, titulado "El reparto del Pacífico", en el primer número de la Revista de Marina, Benjamín Vicuña Mackenna invitaba a tomar posesión de la isla de Pascua y se quejaba entonces de la falta de visión del Estado chileno frente a otros países más proactivos en estas aguas. En este momento, por parte de algunas potencias europeas, en Chile se proclamó la anexión de la Isla de Pascua.

A mediados de 1887 el presidente de Chile entre 1886 y 1891, José Manuel Balmaceda - abogado, periodista y político chileno -, decidió tomar posesión de la Isla de Pascua, para lo cual envió a Policarpo Toro ${ }^{2}$

1 Díaz Meza, Aurelio: Patria Vieja y Patria Nueva. Santiago, Nascimento, 1938-1945.

2 Policarpo Toro Hurtado, fue un militar chileno, conocido por haber sido comandante de la expedición que incorporó la Isla de Pascua a Chile en 1888. 
a negociar con John Norman Brander ${ }^{3}$ y Alexander Salmon, ${ }^{4}$ los entonces propietarios de la isla.

En 1875 Policarpo Toro viaja a bordo de la corbeta O'Higgins hacia la Isla de Pascua en el que sería su primer contacto con ella. Este viaje, de tipo científico, tenía por objeto efectuar un reconocimiento y cartografía de la isla. Sin embargo, la expedición se encontró con habitantes sufriendo de pésimas condiciones de vida, de los malos tratos de otras expediciones que iban en busca de esclavos y riquezas. Esto despertó la humana preocupación de Toro por el destino de la isla y de sus pobladores. Vuelto al continente buscaría el apoyo de personalidades que le apoyasen en su proyecto de anexionar a Chile aquel territorio insular. El gobierno chileno arrendó la isla a una compañía que la convirtió en una gran estancia ovejera, reduciendo a los indígenas a meros empleados de ésta.

En el plano jurídico - político las consecuencias del Acuerdo de voluntades de 1888 y del cumplimiento de la obligación del Pueblo Rapa Nui, fueron las siguientes: el territorio Rapa Nui es incorporado al territorio chileno el 9 de septiembre de 1888, mediante prescripción adquisitiva y como consecuencia de su incumplimiento de ratificar el Acuerdo de Voluntades. Este incumplimiento le permitió al Estado chileno reconocer la anexión de hecho ya no como efecto del Acuerdo de Voluntades, sino

3 Teri'ivaetua pertenecía a la Dinastía Pōmare, presumiblemente heredera cuando el Reino de Tahití fue anexado a Francia en 1880. El 29 de abril de 1884 se casó con Norman Brander sobrino de la reina Marau en Papeete (Polinésia Francesa) - Teri'itonorua Norman Brander hijo de la princesa Titaua Salmon y John Brander. Era el marido de la princesa Teriivaetua. La pareja se divorció el 21 de enero de 1893, después de que la princesa Teriivaetua descubriera que su marido tenía un romance con su tía Alexandria Manihinihi "Pri” Salmon. La Princesa Teriivaetua nunca se volvió a casar y murió en Papeete el 4 de diciembre de 1918, durante el apogeo de la epidemia de gripe que se había extendido desde Europa. Fue enterrada en el cementerio Royal Pōmare en Arue. PIKE EMORY, Kenneth: Historia de la cultura polinesia : ensayos en honor a Kenneth P. Emory. Bishop Museum Press, Honolulu, 1967.

4 Alexander Solomon nació en 1820 en Hastings, Inglaterra, hijo de John Solomon, era comerciante en Piccadilly, y Catherine Polack, y sobrino de Joel Samuel Polack, Alexander era un comerciante inglés que fue el primer judío en residir en Tahití y se convirtió en secretario de la reina Pōmare IV. Alexander Salmon, pertenecía a la familia real tahitiana. Su hija, Alexandria Manihinihi "Pri" Salmon se casó con su sobrino Norman Brander. Si bien es cierto que Salmon nunca maltrató a los pascuenses y representó algo más cercano para ellos, el hecho de ser tahitiano los perjudicó culturalmente, al producirse la inevitable transculturación entre ambos pueblos. Salmon, llegó con un grupo de compatriotas e introdujo palabras y costumbres tahitianas en la cultura Rapa Nui. GOSSLER, Noel: "A queda social e económica de Salmon / Brander Clan do Tahiti”. En: Revista de História do Pacífico. 40, 2, 2005-09. pp.193-212 
como efecto de un acto de ocupación. Stricto sensu, la anexión legal se habría materializado recientemente por medio de la promulgación de la Ley Pascua, Ley No 1.644 de 1966, que crea un departamento en la Provincia de Isla de Pascua y fija normas para la organización y funcionamiento de servicios públicos, a partir de esta fecha se reconocen a los Rapa Nui derechos de ciudadanía y otros como la exención tributaria y el derecho a la tierra, estableciéndose un proceso de regularización para los Rapa Nui.

Desde el punto de vista administrativo, el hecho de reconocer que la Isla de Pascua posee características propias que le han otorgado títulos emblemáticos como Monumento Histórico Nacional, o Patrimonio Cultural de la Humanidad en 1995, impone que se trate a este territorio y su cultura como una comunidad con desafíos importantísimos en todos los ámbitos. Es decir, satisfacer las demandas turísticas y de auto sustentabilidad.

La relación entre el pueblo Rapa Nui y el Estado chileno, comenzó por la anexión del territorio de la Isla de Pascua a Chile a fines del siglo XIX a través de este Acuerdo de voluntades que fue firmado en castellano y Rapa Nui/ tahitiano antiguo. El rey Rapa Nui, Atamu Tekena y el Consejo de Jefes firmaron el acta de cesión de la isla. Ese día, el Capitán Toro convocó a los jefes de las familias nativas al pie de un asta al costado de la iglesia de la pequeña aldea y leyó en español el Acta de Cesión, que fue traducida al pascuense por un isleño. En dicho acuerdo se establecieron las bases de esta relación, constituyéndose en un instrumento fundamental para determinar los derechos territoriales y de libre determinación del pueblo Rapa Nui. La tradición oral Rapa Nui indica que el rey Atamu Tekena tomó en ese momento un pedazo de hierba con tierra agregada a las raíces, entregándole el pasto a los emisarios chilenos y reservando para sí el puñado de tierra. Con ello significaba que se otorgaba la soberanía de la isla al gobierno chileno, pero que los nativos siempre poseerían el derecho natural de propiedad sobre sus tierras. Asimismo, ante el saco de monedas de oro que Policarpo Toro regalaba a Atamu Tekena, el rey lo rechazó diciendo "[...] llévate tu plata, que yo, ni ningún kanaka, hemos vendido terreno alguno". 5

En 1916 la Isla de Pascua fue incorporada a la jurisdicción del Estado chileno, en calidad de Subdelegación al Departamento de Valparaíso; en 1966 pasó a formar parte del Departamento de la Provincia de Valparaíso - Rapa Nui volvió al Estado chileno tras el término del arrendamiento, y,

5 COMISIÓN VERDAD HISTÓRICA Y NUEVO TRATO: Informe Comisión Verdad Histórica y Nuevo Trato. El pueblo Rapa Nui. Santiago, Biblioteca del Congreso Nacional de Chile, 2001. 
desde entonces, el desarrollo del turismo y la revaloración de su patrimonio arqueológico, han marcado una nueva relación entre sus habitantes y el Estado chileno - y en 1982, fue ascendida a Provincia de la V Región.

En 1979, durante la dictadura militar del general Pinochet, se dicta el DL. 2.885, para la regularización de la propiedad de la tierra mediante el otorgamiento de títulos gratuitos de dominio a los poseedores regulares. Una de las medidas contempladas específicamente para la administración de las tierras desde el Fisco a poseedores regulares, aquella que procede de justo título y ha sido adquirida de buena fe, solo incluye las tierras en las que fueron reducidos los Rapa Nui luego de la anexión al estado de Chile, esto es Hanga Roa.

En el año 1993, restaurada la democracia en Chile, se dictó, la denominada Ley indígena, Ley $\mathrm{N}^{\circ} 19.253$ de 1993 sobre "Protección, fomento y desarrollo de los indígenas", la cual establece normas enfocadas en los pueblos originarios de Chile. Esta ley busca reconocer a los distintos pueblos indígenas de Chile como habitantes del territorio desde tiempos precolombinos, es decir, anteriores al establecimiento y dominio de la cultura europea. En base a este reconocimiento se establecen normas para otorgar respeto y protección a la cultura de los pueblos indígenas. Para alcanzar estas metas se forman la Corporación Nacional de Desarrollo Indígena CONADI y la Comisión de Desarrollo de Isla de Pascua CODEIPA - rol fundamental en el desarrollo del territorio-.

La Ley Indígena, en su art. $1^{\circ}$ reconoce a los Rapa Nui como una "etnia", haciéndoles extensivos todos los derechos por ella consagrados a las etnias indígenas y deberes de promoción de derechos que vinculan al Estado. Consagra normas particulares relativas a la etnia Rapa Nui en los artículos $66^{\circ}$ y siguientes. Particularmente, en el artículo $67^{\circ}$ crea la Comisión de desarrollo de Isla de Pascua (CODEIPA), en la que radica una serie de funciones, destacando su participación en la regularización de las tierras de la Isla, para lo que la ley indígena se remite a lo dispuesto en el DL 2.885, utilizando la misma fórmula y restricciones, pero ahora a cargo de la CODEIPA, en reemplazo de la antigua Comisión creada por el DL. 2.885. A fin de cumplir con sus objetivos, este decreto contemplaba la formación de la Comisión de Radicaciones, que es la predecesora legal de la Comisión de Desarrollo de Isla de Pascua (CODEIPA). 
Mientras que la Comisión de Radicaciones se encargaba únicamente del asunto de las tierras en la isla, CODEIPA recibió otras atribuciones. ${ }^{6}$ No solo se ocuparía de regularizar la propiedad de la tierra, sino que también estaría dentro de sus funciones velar por el desarrollo económico, social y cultural del pueblo Rapa Nui.

Consejo de Ancianos Preside esta comisión el Gobernador y actúa como Secretario Técnico, el Jefe de la Oficina de Asuntos Indígenas de Isla de Pascua.

La doctrina contemporánea más relevante es la de Naciones Unidas, con el "Estudio sobre los tratados, convenios y otros acuerdos constructivos entre los Estados y las poblaciones indígenas", encomendado por la Subcomisión sobre Prevención de la Discriminación y Protección de las Minorías (Resolución 1987/17) a M. A. Martínez, Relator Especial, y por la Resolución 1988/56 de la Comisión de Derechos Humanos.

A partir de la caída del régimen autoritario de Augusto Pinochet el año 1989, la pluralidad de realidades culturales en Chile vuelve a visibilizarse para la opinión pública, surgiendo así una serie de movimientos reivindicatorios tanto políticos como académicos que denuncian la

6 Entre las atribuciones de CODEIPA están:

- Proponer al Presidente de la República la destinación de tierras para fines científicos y otros que sean de interés de la comunidad (turismo, áreas de esparcimiento, deportes y similares).

- Formular y desarrollar planes y proyectos de desarrollo que eleven el nivel de vida de la comunidad Rapa Nui y contribuyan a la conservación de su cultura, así como del medioambiente de la isla.

- Colaborar en la administración del Parque Nacional Rapa Nui.

- Colaborar en la conservación y restauración del patrimonio arqueológico Rapa Nui.

- Preparar convenios con personas e instituciones nacionales y extranjeras para conseguir sus objetivos.

Según el Artículo 68 de la "Ley Indígena" (Ley No 19.253), la CODEIPA se compone por:

- Un representante del Ministerio de Desarrollo Social, • Un representante del Ministerio de Educación;

- Un representante del Ministerio de Bienes Nacionales;

- Un representante del Ministerio de Defensa Nacional;

- Un representante de la Corporación de Fomento de la Producción,

- Un representante de la Corporación Nacional Forestal

- Un representante de la Corporación Nacional de Desarrollo Indígena CONADI

- El Gobernador de Isla de Pascua;

- El Alcalde de Isla de Pascua,

- Y 6 miembros electos de la comunidad rapa nui, uno de los cuales debe ser el Presidente del 
urgencia de que el estado democrático reconozca y valide la condición multicultural del país.

En este contexto, y pasada ya una década desde la refundación de la democracia, y en la cual proliferó el debate interétnico y las luchas reivindicatorias, a la vez que desde el mundo académico se comenzó a introducir la presencia del mundo indígena, el año 2001, durante la presidencia de Ricardo Lagos, y mediante la imperativa legal del Decreto Supremo No 19 del 18 de enero del año 2001, se creó la Comisión de Verdad Histórica y Nuevo Trato $^{7}$ - fue un organismo existente en Chile, entre 2001 y 2003, que tuvo por objeto asesorar al presidente de la República en el conocimiento de la visión de los pueblos indígenas del país sobre los hechos históricos de Chile y efectuar recomendaciones para una nueva política de Estado, a fin de avanzar hacia un nuevo trato de la sociedad chilena y su reencuentro con los pueblos originarios.

\section{EL TERRITORIO ${ }^{8}$ Y LA PERTENENCIA ÉTNICA.}

Vivimos en un mundo, en lo cual, los niveles de complejidad regulatoria aumentan día tras día, implicando una especial importancia el Derecho Internacional Privado, que como sistema de normas jurídicas que determinan la jurisdicción competente o la ley aplicable en caso de concurrencia simultánea de dos o más jurisdicciones o de dos o más leyes en el espacio, brinda soluciones a numerosas situaciones provenientes de la interacción humana. Debido a esa transformación, aumentaron los conflictos de normas en el espacio produciendo en algunos casos, reales situaciones de vacío normativo y doctrinal.

Para entender este apartado debemos delimitar los conceptos de jurisdicción y competencia, para tal, se debe recordar que dado a que los Estados latinoamericanos integrados por pueblos indígenas tienen carácter plurilegislativo ratione personae, entre ellos está Chile. Nos deparamos con jurisdicciones diferenciadas dentro de la estructura judicial: jurisdicción especial de cada pueblo indígena que integran el Estado en cuestión, y jurisdicción ordinaria.

$7 \quad$ Vid., Nota 5

8 PEREYRA - UHRLE, Maria: "Easter Island Land Law". En 12. RJP/NZAL Yearbook 11, New Zealand Association of Comparative Law, 2011. p.135 


\section{Actualidades (2020/2021)}

Desde los primeros meses del 2020, la humanidad ha sido afectada por la pandemia causada por el COVID-19, la que ha producido catastróficos efectos a nivel mundial, y donde los pueblos indígenas han sufrido graves impactos, no solo en cuanto a su situación sanitaria y el ejercicio de su derecho a la salud, sino que también la posición de desigualdad social que históricamente han ocupado, los ha llevado a enfrentar grandes afectaciones en el ejercicio de sus derechos económicos, sociales, culturales y ambientales. En este contexto, el pueblo Rapa Nui ha sido un ejemplo en su forma de enfrentar la pandemia, mediante un trabajo articulado de sus liderazgos y el uso de sus conocimientos tradicionales.

En efecto, mediante carta entregada a la Presidenta Michelle Bachelet, el Ariki (Rey) Valentin Riroroko y el representante electo del pueblo Rapa Nui ante el Estado de Chile (Conadi), Rafael Tuki, presentaron los HONUI: asamblea de representantes de las 36 familias ancestrales descendientes de la civilización Maori Rapa Nui. La idea de constituir los HONUI, o consejo de clanes, tiene muchos antecedentes inconclusos. Pero tras el conflicto con CONAF por el control del "parque nacional" - iniciado en marzo de 2015- los clanes se tomaron en serio la conformación de un espacio de representación del pueblo Rapa Nui. a asamblea de clanes decidirá respecto a la administración del parque, migración, parque marino y todo el desarrollo de la isla en conjunto con otras instituciones como CODEIPA. En todo este proceso las familias han tenido que mirar hacia atrás y aprender su árbol genealógico, y muchos jóvenes hoy se esmeran en aprender su tumu, su aka y su haka ara, por lo que ha sido un proceso muy enriquecedor. El sistema de HONUI fue un sistema que existía antiguamente un tipo la organización de los clanes y autoridad se reflejaba a través del hermano mayor. Hoy se pretende rescatar este sistema adaptándolo a los nuevos tiempos y concepciones, a través de voceros electos.

En primer lugar, catorce días después de que se detectara el primer caso de COVID-19 en Chile, el 17 de marzo, y ante la negativa del Gobierno de Chile de tomar medidas de protección al territorio Rapa Nui, el pueblo de forma autónoma y actuando en unidad de sus órganos de liderazgos administrativos y políticos, tales como la Asamblea de Clanes HONUI, el Parlamento Rapa Nui y la Municipalidad de Rapa Nui, tomaron la decisión de cerrar sus fronteras.

De recordar que, mediante carta entregada a la Presidenta Michelle Bachelet, el Ariki (Rey) Valentin Riroroko y el representante electo del 
pueblo Rapa Nui ante el Estado de Chile (Conadi), Rafael Tuki, presentaron los HONUI: asamblea de representantes de las 36 familias ancestrales descendientes de la civilización Maori Rapa Nui. La idea de constituir los HONUI, o consejo de clanes, tiene muchos antecedentes inconclusos. Pero tras el conflicto con CONAF por el control del parque nacional - iniciado en marzo de 2015 - los clanes se tomaron en serio la conformación de un espacio de representación del pueblo Rapa Nui. La asamblea de clanes decidirá respecto a la administración del parque, migración, parque marino y todo el desarrollo de la isla en conjunto con otras instituciones como CODEIPA. El sistema de HONUI fue un sistema que existía antiguamente un tipo la organización de los clanes y autoridad se reflejaba a través del hermano mayor. Hoy se pretende rescatar este sistema adaptándolo a los nuevos tiempos y concepciones, a través de voceros electos.

En Marzo 2021, se tomó el aeropuerto a través de protestas para evitar que siguieran llegando vuelos comerciales sin ningún tipo de control, única forma de ingreso a Rapa Nui, separada por 3.700 kilómetros de la costa de Chile continental y que no cuenta con profesionales e infraestructura sanitaria necesarios para la atención médica normal de la población y mucho menos para enfrentar una pandemia de estas características.

El 23 de marzo de 2020, se confirma el primer caso de COVID-19 en la isla, que se contagió a través de un turista que ingresó precisamente los días en que se solicitaba al gobierno de Chile cancelar los vuelos a Rapa Nui.

El 27 de marzo, finalmente, el Gobierno de Chile decreta cuarentena en la Isla de Pascua, debido a la presencia de contagiados (cinco) y a la imposibilidad de parar su transmisión.

El 5 de abril, el gobierno anuncia que se pone término a la cuarentena en Rapa Nui, sin que hayan cambiado las circunstancias sanitarias, sin previa consulta a las autoridades locales y sin mediar ninguna consideración con lo distintas y particulares que son las características de este territorio indígena. Esta situación obligó a presentar un recurso constitucional ante las Cortes superiores de justicia de Chile, pidiendo que se anule esta medida que los dejaba desprotegidos como pueblo indígena. El recurso fue rechazado por los tribunales chilenos, bajo fundamento de encontrarse el país en un estado de excepción constitucional, que permite al gobierno tomar todo tipo de medidas, tales como limitar los derechos de sus habitantes.

Ante este abandono del gobierno, las autoridades Rapa Nui, alineadas con su alcalde, decidieron invocar la ley ancestral de su pueblo a través de una medida llamada tapu, concepto que constituye una orden sagrada que 
se basa en la convivencia y en el respeto a las normas de la naturaleza y que consistía en realizar una cuarentena total y voluntaria en todo el territorio de Rapa Nui. Toda la comunidad acató responsablemente la medida y con el esfuerzo de su pueblo lograron controlar la enfermedad en su territorio.

Desde la cancelación de los vuelos a Isla de Pascua, gracias a la presión de sus autoridades propias, quedaron cientos de miembros del pueblo Rapa Nui atrapados en el territorio continental de Chile. Ante esta situación se trabajó de manera local y autónoma sin ningún tipo de ayuda gubernamental en un plan de "Retorno seguro a Rapa Nui" para poder traer de vuelta a sus miembros y evitar volver a ingresar el virus del COVID-19 a la isla. Este plan contemplaba un protocolo autónomo y un equipo de acción que involucró a toda la comunidad, que resultó ser un éxito, logrando a la fecha haber repatriado a más de mil miembros de la comunidad, manteniéndose como territorio libre de COVID-19.

Por su parte, los impactos socio-económicos se han vivido fuertemente en Rapa Nui, puesto que la economía local se sustenta únicamente en la industria del turismo, la que ha sido la principal afectada por esta pandemia. Hasta antes de marzo de 2020, a Rapa Nui llegaban dos vuelos comerciales diarios, con una tasa de visitación de más de 120.000 turistas al año, lo que provocó el desplome de la economía y un estado total de desempleo.

A su vez, gran parte del alimento y abastecimiento de Rapa Nui se realiza por vía aérea. Por eso, al no tener vuelos con turistas (únicamente de carga), se han elevado tremendamente los precios de los productos, lo que, sumado a la falta de ingresos, ha perjudicado a gran parte de la comunidad. Cabe destacar que el Gobierno de Chile se ha negado a enviar alimentos o subsidios para abastecer al pueblo Rapa Nui, pese a las varias solicitudes que han realizado los líderes locales.

Ante este desolador escenario, el pueblo Rapa Nui se volcó a producir sus propios alimentos mediante el cultivo de vegetales y la pesca dentro de sus familias, lo que ha permitido enfrentar de manera autónoma y autosustentable todos estos meses de pandemia y cierre total de fronteras.

Así también, la Municipalidad de Rapa Nui creó un inédito programa de generación de empleos destinando todo su presupuesto a entregar trabajo a más de 700 habitantes basado en cinco ejes: impulsar seguridad alimentaria, renovar y mantener espacios públicos, resguardar y promover los valores culturales, apoyar a emprendedores, mentores y contratistas locales y fortalecer el sistema de información, seguridad y protección social. A su vez, se empleó a gran parte de los trabajadores de la Comunidad 
Indígena Maú Henua, órgano administrador del Parque Nacional Rapa Nui que perdieron su fuente de trabajo.

\section{CONCLUSIONES.}

El Acuerdo de Voluntades constituye un tratado internacional que representa la participación consensual del Pueblo Rapa Nui y el Estado de Chile en una relación jurídica.

El Pueblo Rapa Nui realizó diversos intentos y gestiones para lograr el cumplimiento de las obligaciones por parte de Chile, interpelando al Estado chileno, pero éste no respondió, o si lo hizo fue por medio de la represión y el olvido.

La Iglesia Católica fue instigadora del proceso de anexión de la isla, desempeñando un rol, a lo menos, ambivalente. Con todo, participó en la violación de los derechos humanos del Pueblo Rapa Nui y fue uno de los agentes promotores de la forma de propiedad privada sobre la tierra en te pito o te henua - es una enorme piedra redonda que representa el ombligo del mundo para los isleños. El significado de pito se traduce al portugués como útero, centro y ombligo, mientras que henua es tierra, territorio y universo, que también constituye una forma de vulneración de derechos humanos.

El territorio Rapa Nui es agregado legalmente al territorio chileno mediante prescripción adquisitiva y como consecuencia de su incumplimiento de ratificar el Acuerdo de Voluntades. Este incumplimiento le permitió después al Estado chileno reconocer la anexión de hecho, ya no como efecto del Acuerdo de Voluntades, sino como efecto de un acto de ocupación. Y esta ocupación pudo luego ser justificada gracias al proceso de retrogresión, que pareciera haberse concentrado, primero y fundamentalmente, en eliminar la capacidad de gobierno del Pueblo Rapa Nui. Con la pérdida de sus tierras, finalizó exitosamente la retrogresión. La prescripción adquisitiva vino por añadidura. La anexión legal se verificó con la promulgación de la "Ley Pascua".

El Estado de Chile debe compensar material y espiritualmente al Pueblo Rapa Nui por los daños causados. Las bases de la compensación deben ser establecidas sobre criterios consensuados con el propio Pueblo Rapa Nui, no excluyendo a ningún sector u organización de él, y con absoluto respeto a su cultura. Para ello debe constituirse una Comisión de Reparación para el Pueblo Rapan Nui, con participación de Naciones Unidas. La 
compensación debe a lo menos considerar: restitución íntegra de las tierras en la forma que el Pueblo Rapa Nui decida; la restauración y conservación del patrimonio cultural material; la recuperación y traslado a la isla del patrimonio cultural material que se encuentra en Chile continental y en museos y similares del mundo; pensiones de reparación para víctimas y familiares de violación de derechos humanos; investigación de situaciones específicas, como lo sucedido con el Ariki Riroroko, Clementina Hito, Daniel María Teave y otros. Se sugiere al Pueblo Rapa Nui registrar el Acuerdo de Voluntades según la institucionalidad que ha creado Naciones Unidas para tales efectos. Es de esperar que la actitud chilena no se altere, que se profundice la política descentralizada de la isla, y que se continúe promoviendo la recuperación de la isla para sus habitantes originarios.

En la experiencia de Rapa Nui debe destacarse el cambio radical de actitud experimentado por el Estado de Chile frente a la etnia. Si durante la mayor parte del siglo XX los Rapa Nui fueron marginados y dominados políticamente por la maquinaria política colonial, últimamente Chile ha mostrado apertura hacia la perspectiva indígena al punto de estar comprometido en la actualidad en un modelo de gestión participativo completamente inédito para el país. En fin, el nuevo modelo parece poner definitivamente fin a la gestión centralizada, autoritaria y etnochilena de la isla y avanzar decididamente hacia una rapanuización del territorio insular, en un doble sentido: por una parte, hacia una cada vez mayor injerencia Rapa Nui en la política de Isla de Pascua; y, por la otra, hacia la localización de la gestión atribuyendo a la CDIP importantes facultades políticas que deberán conciliarse con las propias de la municipalidad local.

A pesar de ser uno de los grupos más vulnerables y marginados del mundo, los pueblos indígenas han demostrado ser fuertes, y capaces de organizarse y defenderse. Aún ocupan muchos de sus territorios ancestrales, celebran sus culturas únicas y luchan por mantenerlas, y actúan como los guardianes de la mayor parte de la diversidad biológica y cultural del mundo. Los pueblos indígenas son una parte integral de la sostenibilidad y gestión correcta de los recursos naturales; sus conocimientos y entendimiento de nuestro planeta constituyen una parte clave de las soluciones necesarias para conseguir un futuro más neutral, razonable y sostenible para toda la humanidad.

En 2021, en el marco de la Consulta Indígena para la Modificación y/o Derogación de los artículos 13 y 14 de la Ley Pascua. Por más de 10 años, diversas organizaciones sociales levantaron la necesidad de trabajar en la 
protección de los derechos de niños y mujeres, que se ven vulnerados por los artículos 13 y 14 de esta ley, que establecen beneficios carcelarios para los responsables de delitos graves de violación y abuso sexual cometidos en la isla.

Esta pandemia ha dejado en evidencia la situación de abandono estatal que enfrenta el pueblo Rapa Nui, donde los derechos básicos como acceso a telecomunicaciones, conexión con el exterior, abastecimiento y traslado de carga, se encuentran totalmente supeditados a empresas privadas y transnacionales. Otro gran problema ha sido la falta de autonomía que tiene su pueblo sobre su territorio, lo que impide a sus autoridades tradicionales tomar decisiones para el cuidado de su comunidad, viéndose obligados a depender de decisiones de autoridades gubernamentales que se ubican a miles de kilómetros de distancia en el continente y que ignoran totalmente la realidad Rapa Nui. El ejercicio a los derechos a la libre determinación y derechos territoriales del pueblo Rapa Nui sobre su propia isla, se han vuelto una importante necesidad y constituyen la prioridad de las demandas de su pueblo en sedes nacionales e internacionales.

\section{BIBLIOGRAFÍA.}

AYLWIN OYARZÚN, José: Derechos humanos y pueblos indigenas: tendencias internacionales y contexto chileno. IWGIA, Universidad de la Frontera, Temuco, 2004.

ANDUEZA, Pablo: "Mediación en una sociedad multicultural: El modelo de cogestión en Rapa Nui”. En: ¿Hay patria que defender? La identidad nacional frente a la globalización. CED, Santiago, 2000

- "Hacia el reconocimiento de los derechos políticos de los pueblos originarios: El modelo de cogestión en Isla de Pascua". En: Estudios Atacameños. Instituto de Investigaciones Arqueológicas y Museo, Universidad Católica del Norte, $\mathrm{N}^{\mathrm{o}}$ 19, 2000.

BENGOA, José: La memoria olvidada: historia de los pueblos indigenas de Chile. Cuadernos Bicentenario, Presidencia de la República, 2004

BOURDON - VIANE, G.: Compendio de Derecho Internacional Público. Traducido por Eduardo Phillips. Imprenta Mejía. Santiago, 1897. 
CORVALÁN, Javier: Educación en Rapa Nui: Sociedad y escolarización en Isla de Pascua (1914-2014) Ediciones Universidad Alberto Hurtado, Santiago, 2015.

DÍAZ MEZA, Aurelio: Patria Vieja y Patria Nueva. Santiago, Nascimento, 1938-1945.

GOSSLER, Noel: "A queda social e económica de Salmon / Brander Clan do Tahiti”. En: Revista de História do Pacífico, 40, 2, 2005.

HIDALGO, Jorge et al (Eds.): Etnografia Sociedades indígenas contemporáneas y su ideología, Editorial Andrés Bello, Santiago, 1996.

MARTÍNEZ, José: Estudio del problema de la discriminación contra las poblaciones indigenas. Naciones Unidas, Nueva York, 1987.

MCCALL, Grant: Rapanui. Tradición y sobrevivencia en Isla de Pascua. Traducción Betty. Haoa. Easter Island Foundation, Los Osos, California, 1998.

PEREYRA - UHRLE, Maria: "Easter Island Land Law". En: 12. RJP/ NZAL Yearbook 11. New Zealand Association of Comparative Law, 2011.

PIKE EMORY, Kenneth: Historia de la cultura polinesia : ensayos en honor a Kenneth P. Emory. Bishop Museum Press, Honolulu, 1967

PORTEOUS, John Douglas: The Modemization of Rapanui, Easter Island Studies: Contributions to the History of Rapanui in Memory of William T. Mulloy, S.R. Fisher. Ed., 1993.

RIBERA, Teodoro: Los pueblos indígenas y su reconocimiento constitucional en Chile. Centro de Estudios Públicos, Santiago, 2002.

ROCHNA - RAMÍREZ, Susana: La propiedad de la tierra en Isla de Pascua. CONADI, Santiago, 1996

SEPÚLVEDA, Milo: Rapa Nui, 100 años: reportaje a la última frontera. Ediciones Aku-Aku, Easter Island, 1990.

VERGARA, Víctor: La Isla de Pascua. Dominación y Dominio. Publicaciones de la Academia Chilena de la Historia. Santiago, 1939.

WITKER, Jorge y VELASCO, Rogelio: Metodología Jurídica. McGrawHill Interamericana Editores, S.A. de C.V. Segunda Edición. México, 2002 


\section{Otras consultas}

COMISIÓN VERDAD HISTÓRICA Y NUEVO TRATO: Informe de la Comisión Verdad Histórica y Nuevo Trato con los Pueblos Indígenas. Gobierno de Chile. Ministerio de Planificación, Santiago, 2009

COMISIÓN PROVINCIAL DE VERDAD HISTÓRICA Y NUEVO TRATO DE ISLA DE PASCUA: La verdad histórica de rapanui y Planteamiento final. Documento de Trabajo, Comisión Verdad Histórica y Nuevo Trato. CVHNT/ GTRN/2002/056, 2002, Hanga Roa.

CONSEJO DE JEFES RAPA NUI, A. HOTUS y otros: Te Mau Hatu'o Rapa Nui. Los soberanos de Rapa Nui. Pasado, presente y futuro. Editorial Emisión y Centro de Estudios Políticos Latinoamericanos Simón Bolívar, Primera Edición. Santiago, 1988 